\title{
The clinicopathological features of drug-induced acute kidney injury-a single-center retrospective analysis
}

\author{
Yu Cui ${ }^{1,2,3 \#}$, Yi Yang ${ }^{1,2,3 \#}$, Wenhua Lei ${ }^{1,2,3}$, Xiabing Lang ${ }^{1,2,3}$, Jianghua Chen $^{1,2,3}$
}

${ }^{1}$ Kidney Disease Center, The First Affiliated Hospital, College of Medicine, Zhejiang University, Hangzhou, China; ${ }^{2}$ Key Laboratory of Kidney Disease Prevention and Control Technology, Zhejiang Province, National Key Clinical Department of Kidney Diseases, Institute of Nephrology, Zhejiang University, Hangzhou, China; ${ }^{3}$ The Third Grade Laboratory under the National State, Administration of Traditional Chinese Medicine, Hangzhou, China

Contributions: (I) Conception and design: Y Yang, J Chen; (II) Administrative support: J Chen; (III) Provision of study materials or patients: Y Cui; (IV) Collection and assembly of data: Y Cui, W Lei, X Lang; (V) Data analysis and interpretation: Y Yang, Y Cui, X Lang; (VI) Manuscript writing: All authors; (VII) Final approval of manuscript: All authors.

\#These authors contributed equally to this work.

Correspondence to: Yi Yang; Jianghua Chen. Department of Nephrology, the First Affiliated Hospital, College of Medicine, Zhejiang University, Hangzhou 310003, China. Email: yangyixk@zju.edu.cn; chenjianghua@zju.edu.cn.

Background: This study aimed to analyze changes to the drug spectrum and clinicopathological features of drug-induced acute kidney injury (AKI) with recent medication habits changes.

Methods: A retrospective analysis of the characteristics of patients diagnosed with drug-induced AKI from January 2012 to October 2016 period at the First Affiliated Hospital of the Medical College of Zhejiang University was conducted.

Results: Between January 2012 and October 2016, 909 patients were diagnosed with AKI. Of these, 228 were diagnosed with drug-related AKI were engaged in this study, including 51 who underwent renal biopsies, 74 treated with antibacterial and antiviral drugs, and 63 who received nonsteroidal antiinflammatory drugs (NSAIDs), and 17 who were treated with Chinese herbal medicine. AKI was most frequently associated with antibiotics and antiviral drugs, including cephalosporins, acyclovir, azithromycin, clindamycin, and levofloxacin. In those who underwent renal biopsy, 12 patients were diagnosed with allergic interstitial nephritis, 19 with interstitial nephritis, 8 with renal tubular epithelial cell injury, 2 with minimal change nephropathy, 2 with IgA nephropathy, and 2 with mild mesangial hyperplasia with glomerulosclerosis. The mean follow-up time was 437 days, ranging from 3 to 2,756 days. Among 228 patients, 165 recovered completely, 4 recovered partially, 8 did not recover, and 51 were lost to follow-up after discharge.

Conclusions: The three main contributors to drug-induced AKI were antimicrobial agents, NSAIDs, and Chinese herbal medicines. The age distribution of the three different drug-induced AKI groups was significantly different. Allergic interstitial nephritis, interstitial nephritis, and tubular epithelial cell injury were the main pathological manifestations of drug-induced AKI. The novel predictive nomogram achieved a good performance of prediction recovery within 2 weeks in drug-induced AKI patients.

Keywords: Drug-induced acute kidney injury; nephrotoxicity; renal pathology; prognosis; nomogram

Submitted May 10, 2020. Accepted for publication Nov 06, 2020.

doi: $10.21037 /$ atm-20-3826

View this article at: http://dx.doi.org/10.21037/atm-20-3826 


\section{Introduction}

As the excretory organ for most drugs, the kidney is susceptible to drug-induced acute kidney injury (AKI). This is evidenced by the fact that $15 \%$ of in-hospital and 40 $60 \%$ of intensive care unit patients with AKI are recognized as drug-induced and are associated with high mortality (1-6). Although drug-induced AKIs are frequently reported, the distribution of drugs causing AKIs has not been clearly identified. It is important to study the drug spectrum of drug-induced AKI because of the severity of this disease and the spectrum of drugs involved $(7,8)$.

Drug-induced AKI accounts for $19 \%$ of AKIs in a hospital setting (9). Nephrotoxicity is most commonly associated with injury in the tubulointerstitial compartment, manifesting as either acute tubular injury or acute interstitial nephritis (10). Multiple reports also highlight the potential for drug-induced glomerular diseases, including direct cellular injury and immune-mediated injury. It is critical to recognize drug-induced nephropathies and rapidly discontinue the offending agents to maximize the likelihood of recovering renal function.

Brown et al. (11), demonstrated a decrease in survival in almost 2,000 postoperative AKI patients with a longer duration of AKI, especially when it lasted longer than 7 days. Similarly, Han et al. reported an inverse relationship between AKI's survival and duration in critically ill patients (12). However, few studies focus on renal recovery from AKI or drug-induced AKI in patients who do not need renal replacement therapy (RRT) (13). Studies also showed that early nephrology follow-up for AKI after hospitalization might improve survival (14). Therefore, it is of great clinical value to understand the recovery rate and recovery time of AKI patients and establish a model for the early assessment of short-term renal function recovery based on fewer and easily accessed available clinical indicators.

Our research focused on changes to and characteristics of the drug spectrum of drug-induced AKI patients, as well as pathological characteristics of the kidney, recovery rate, recovery time, and risk factors for renal function recovery in 2 weeks, and we established a nomogram for predicting recovery within 2 weeks according to the risk factors.

We present the following article following the STROBE reporting checklist (available at http://dx.doi. org/10.21037/atm-20-3826).

\section{Methods}

The study was conducted following the Declaration of
Helsinki (as revised in 2013). The research ethics committee approved the study of the First Affiliated Hospital, College of Medicine, Zhejiang University (NO.: 2019-1363). All patients provided written informed consent. Patients were clinically diagnosed with drug-induced acute renal injury between January 2012 and October 2016, and their serum creatinine levels after discharge were included in the study.

Drug exposure was classified as a history of receiving medications before developing an AKI. Patients were excluded if they had a clear alternative diagnosis explaining their AKI (e.g., hemolytic uremic syndrome, transplant rejection, or acute glomerulonephritis). Although volume depletion is an independent risk factor for AKI, patients with a history of volume depletion in the absence of sepsis or multi-organ failure were retained because it is likely that volume depletion increases the likelihood of AKI resulting from the use of nonsteroidal anti-inflammatory drugs (NSAIDs) in treatment. Patient data contained the following items: age, sex, race, kind of drugs, and serum creatinine (SCr) before, during, and after AKI.

AKI was defined as an increase in serum creatinine of $0.3 \mathrm{mg} / \mathrm{dL}$ in $48 \mathrm{~h}$, a $>50 \%$ increase in serum creatinine over $7 \mathrm{~d}$, or a urine output of $<0.5 \mathrm{~mL} / \mathrm{kg}$ per h for $>6 \mathrm{~h}$. If baseline creatinine was not available, AKI was defined as SCr exceeding 1.5 times the age-adjusted reference value $(15,16)$. The calculation formula was as follows $(17)$ :

$$
\begin{aligned}
\text { Serum creatinie } & =\left\{75 /\left[186 \times\left(\text { age } e^{-0.203}\right)\right.\right. \\
& \times(0.742 \text { if female }) \times(1.21 \text { if black }]\}^{-0.887}[1]
\end{aligned}
$$

Drug-induced AKI was defined as the AKI retained after excluding the following conditions according to the history or pathological results: (I) Patients with a clear history of chronic kidney disease; (II) AKI caused by hemodynamic instability due to blood loss during trauma or operation; (III) AKI caused by septic shock due to infection or sepsis; (IV) AKI caused by obstructive factors, such as calculi and tumor compression of the ureter; (V) AKI caused by other reasons, such as urinary tract infection, lymphoma, or hepatorenal syndrome.

The recovery extent of renal function at hospital discharge was classified into three levels: (I) total ( $\mathrm{SCr}$ $\leq 1.4 \mathrm{mg} / \mathrm{dL}$ ); (II) partial ( $\mathrm{SCr}>1.4 \mathrm{mg} / \mathrm{dL}$ and lower than the maximum SCr reached); and (III) absence, if the $\mathrm{SCr}$ was similar to the highest level observed during the ARF episode or if the patient still needed renal replacement therapy (18).

Renal biopsy was performed in 51 patients, and the 

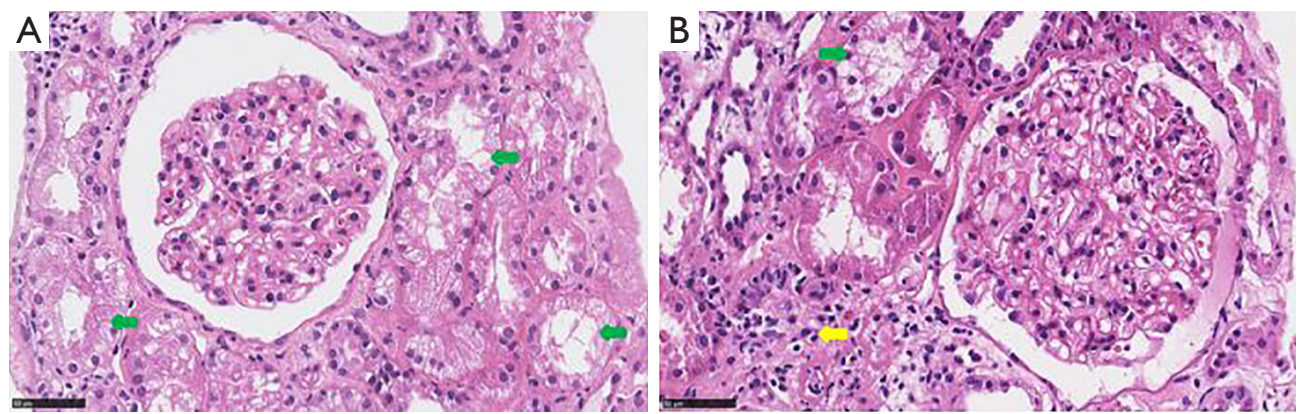

Figure 1 High-power photomicrograph showing (A) relatively normal glomeruli and severe acute tubular injury in the interstitial area (Hematoxylin \& Eosin); (B) severe acute tubular injury and mononuclear cell infiltration with interstitial edema (Hematoxylin \& Eosin).

tissue was then evaluated with light microscopy with hematoxylin and eosin, periodic acid-Schiff (PAS), Masson trichrome, and periodic acid-silver methenamine. Direct immunofluorescence of frozen tissue was used to detect IgG, IgA, IgM, C3, and C1q (Beijing, China). Pathological images revealed that 51 patients were diagnosed with allergic interstitial nephritis, interstitial nephritis, renal tubular epithelial cell injury, minimal change nephropathy, IgA nephropathy, and mild mesangial hyperplasia with glomerulosclerosis. Renal ultrastructure was examined using an electron microscope.

\section{Statistical analysis}

Normally distributed data are presented as means $\pm \mathrm{SD}$, and groups were compared using 2 independent sample $t$-tests or variance analysis (ANOVA). Multivariate analysis was performed using a binary logistic regression method, and the $\mathrm{P}$ value was set $<0.05$. A nomogram was also constructed based on the significant factors to predict drug-induced AKI recovery within 2 weeks, with $\mathrm{P}$ value $<0.05$ considered statistically significant. All statistical analyses were performed using $\mathrm{R}$ software, and the discriminative ability of the model was assessed by the concordance index (C-Index) with the goodness of fit shown in the calibration plot.

\section{Results}

\section{Patient characteristics and pathological characteristics}

Between January 2012 to October 2016, 909 patients were diagnosed with AKI, and 228 of these diagnosed with druginduced AKI based on the exclusion criteria were included in this study, including 51 individuals who underwent renal biopsies. A total of $33.8 \%$ of patients (77/228) were female, and the median age at diagnosis was 53-year (ranging from 16-82 years). Figure 1 shows the age distribution of the 228 patients in this study. Complete recovery was seen in 165 patients $(72.4 \%), 4$ patients (1.8\%) recovered partially (at more than 3 months follow-up time, creatinine was between 124-177 $\mu \mathrm{mol} / \mathrm{L}$ ), 8 patients $(3.5 \%)$ did not recover (at more than 3 months follow-up time, creatinine was still higher than $177 \mu \mathrm{mol} / \mathrm{L}$ ), and 51 were lost to follow-up (most of these were not followed up after discharge after showing decreasing creatinine levels). The average followup time was 437 days, with the longest 2,756 days.

The three most common causes of drug-induced AKI were grouped and analyzed. The antimicrobial agentsinduced AKI group's average age was 53.5 \pm 13.7 -year, of the NSAIDs-induced AKI group 45.6 \pm 17.6 -year, and the Chinese herbal medicine-induced AKI group 61.5 \pm 15.0 -year. As shown in Table 1, there were significant differences between both of the two groups $(\mathrm{P}<0.05)$.

Of the 228 patients, 51 individuals underwent renal biopsy. There were 19 cases of acute interstitial nephritis, 12 of allergic interstitial nephritis, 8 of acute tubular injury, 2 of minimal change nephropathy, 2 of IgA nephropathy, 2 of mild mesangial hyperplasia with glomerulosclerosis, and 1 case each of granulomatous inflammation, tubular calcium salt deposition, focal nephritis, chronic interstitial nephritis, proliferative sclerosing nephritis and vasculitis with glomerulosclerosis, and crescent formation respectively. Immunofluorescence shows the presence of IgA, IgM, and $\mathrm{C} 3$, and 18 cases were negative.

There were 21 cases of acute renal injury related to antimicrobial agents, 14 related to nonsteroidal antiinflammatory drugs, and 6 cases related to Chinese herbal medicine, while the other 9 cases related to multiple drugs, including RAS blockers, albumin, and colchicine. Of the 21 cases of AKI caused by antimicrobial agents, 9 
Table 1 Characteristics and pathology of included patients

\begin{tabular}{|c|c|c|c|c|}
\hline Characteristics & Group 1 & Group 2 & Group 3 & Group 4 \\
\hline Male, n (\%) & 38 (51.35) & $51(80.95)$ & $8(47.06)$ & $54(72.97)$ \\
\hline Age (yrs) & $53.5 \pm 13.7^{\star}$ & $45.6 \pm 17.6^{\star}$ & $61.5 \pm 15.0^{*}$ & $53.3 \pm 15.3$ \\
\hline $\operatorname{Crmax}(\mu \mathrm{mol} / \mathrm{L})$ & $576.2 \pm 398.0$ & $437.7 \pm 288.9$ & $438.3 \pm 250.1$ & $481.7 \pm 337.9$ \\
\hline \multicolumn{5}{|l|}{ Pathology } \\
\hline Acute interstitial nephritis, $n$ & 9 & 7 & 2 & 1 \\
\hline Allergic interstitial nephritis, $n$ & 5 & 1 & 1 & 5 \\
\hline Acute tubular injury, $\mathrm{n}$ & 2 & 2 & 1 & 3 \\
\hline
\end{tabular}

Data are expressed as either mean \pm SDE or as number and percentage. Group 1 = Antimicrobial agents-induced AKI, Group $2=$ NASIDsinduced AKI, Group 3 = Chinese herbal medicine-induced AKI, Group 4 = Other drugs-induced AKI. * $\mathrm{P}<0.05$. Crmax, maximum serum creatinine; CR, complete recovery; AKI, acute kidney injury.

had acute interstitial nephritis, 5 had allergic interstitial nephritis, 2 had an acute tubular injury, and 5 had IgA glomerulonephritis with crescents, mild mesangial hyperplasia, and sclerosis, vasculitis changes with sclerosis and crescent formation, calcium deposition in tubules, or focal glomerulonephritis (Figure 1). Of the 14 cases of NSAIDs-induced AKI, 7 had acute interstitial nephritis, 1 had allergic interstitial nephritis, 2 had an acute tubular injury, 2 had small lesions, 1 had IgA nephritis with sclerosis, and 1 had mild mesangial hyperplasia with hardening. There were 6 cases of patients with AKI caused by Chinese herbal medicine, including 1 case of acute interstitial nephritis, allergic interstitial nephritis, chronic interstitial nephritis, granulomatous inflammation, renal tubular epithelial injury, and proliferative sclerosing glomerulonephritis (Table 1).

The maximum SCr of the drug-induced AKI patients was $496.9 \pm 343.8 \mu \mathrm{mol} / \mathrm{L}$; of the antimicrobial agents-induced AKI group, this was $576.2 \pm 398.0 \mu \mathrm{mol} / \mathrm{L}$; of the NSAIDsinduced AKI group, this was $437.7 \pm 288.9 \mu \mathrm{mol} / \mathrm{L}$; and of the Chinese herbal medicine-induced AKI group, this was $438.3 \pm 250.1 \mu \mathrm{mol} / \mathrm{L}$. There were no significant differences in different groups nor different kinds of antimicrobial agents (Figure 2).

\section{Drug analysis}

Of the 228 enrolled patients, AKI was caused by antimicrobial agents in 74 cases (32.46\%), by NSAIDs in 63
(27.63\%), and by Chinese herbal medicines in 17 (7.46\%). There were 31 cases of acute renal injury that may have been caused by a variety of drugs taken before the injury that could not be confirmed, accounting for $13.60 \%$ of the total cases, and 14 cases showed a history of unspecified drug used, accounting for $6.14 \%$ of the total number of cases. The remaining cases of drug-induced AKI were caused by renin-angiotensin system inhibitors (RASI), anticancer drugs, immunosuppressants, proton pump inhibitors, colchicine, allopurinol, lipid-lowering drugs, diet pills, albumin, 5-aminosalicylic acid, and other drugs. Overall, the most common antibacterial drugs that induced AKI were $\beta$-lactam antibiotics, acyclovir, clindamycin, azithromycin, and levofloxacin, but not aminoglycosides (Figure 3).

\section{Progress}

The average follow-up time was 437 days, and the longest was 2,756 days. Among the 228 patients, 165 (72.4\%) recovered completely, 4 (1.8\%) recovered partially, 8 (3.5\%) did not recover, and 51 were lost to follow-up (most of these were not followed up after discharge as they showed decreasing creatinine). The renal function recovery rate of drug-induced AKI patients is shown in Figure 4.

A prognostic nomogram for the early recovery of druginduced AKI patients was constructed using multivariate logistic regression, and points were assigned to the identified factors according to their regression coefficients. 

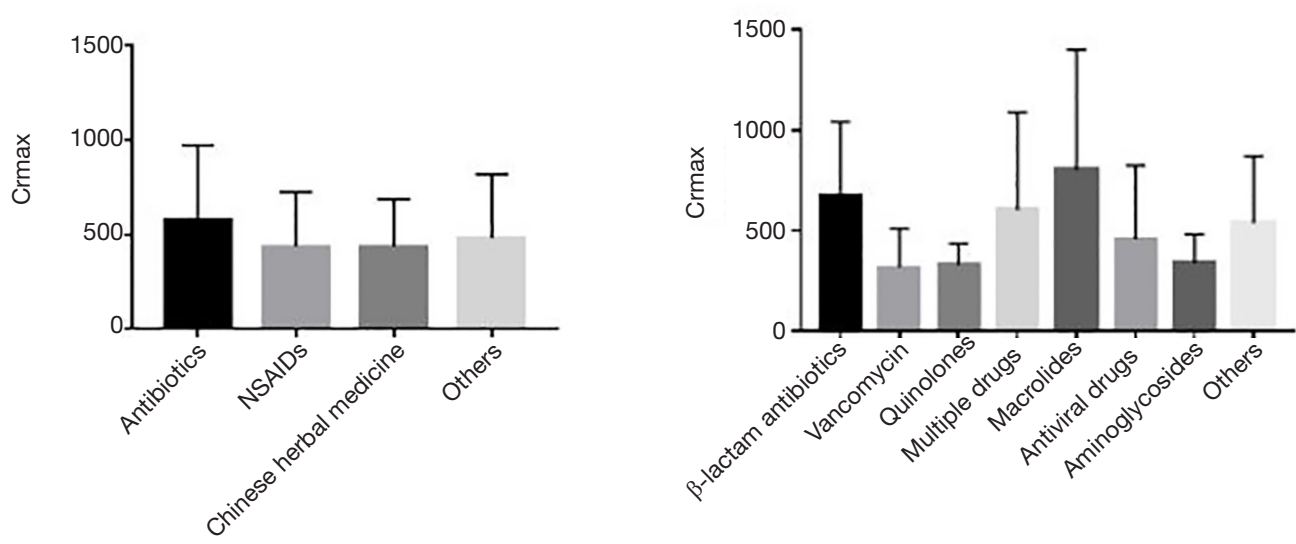

Figure 2 Maximum serum creatinine of different drugs.

Pharmaceutical agents causing AKI

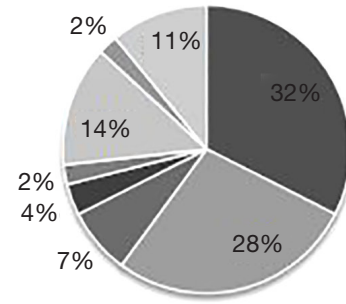

$$
\begin{aligned}
& \text { " Antimicrobial agents } \\
& \text { " NSAIDs } \\
& \text { " Chinese medicine } \\
& \text { " RASI } \\
& \text { " Chemotherapeutic agents } \\
& \text { " Multiple drugs } \\
& \text { "CNI } \\
& \text { "Others }
\end{aligned}
$$

Figure 3 Pharmaceutical agents causing AKI. AKI, acute kidney injury.

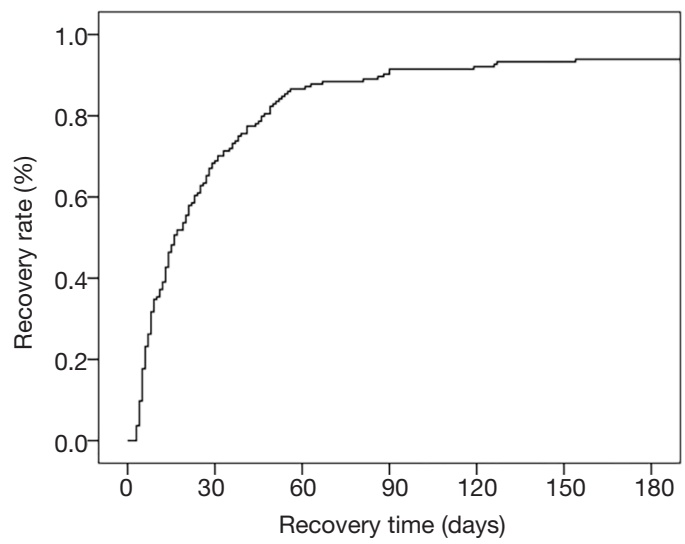

Figure 4 The progress of drug-induced AKI. AKI, acute kidney injury.

As shown in the nomogram, by summing the total score and locating the score on the total point scale, the recovery of drug-induced AKI in two weeks can be predicted for individuals according to the kind of drug used and the maximum SCr of the patient (Figure 5).

To reduce overfitting bias and evaluate the model's discrimination, a bootstrapping technique with 1,000 resamples qualified by Harrell's C statistic concordance index (the Harrell C-index) was performed, which was more accurate than dividing the data into two parts when the dataset was small. In our study, the calibration plot showed excellent accordance between the actual observations of drug-induced AKI and the nomogram prediction (Figure 6).

\section{Discussion}

In a previous study of unplanned hospitalizations caused by adverse drug reactions in older US war-veterans, antibiotics, loop diuretics, ACE inhibitors, and NSAIDs were identified as the main pharmacologic classes (19). More recently, Handler et al. found that the vast majority of drug-associated AKI cases in nursing home residents were related to the use of diuretics, ACE inhibitors, ARBs, and antibiotics (20). A 2017 French study showed that two or 


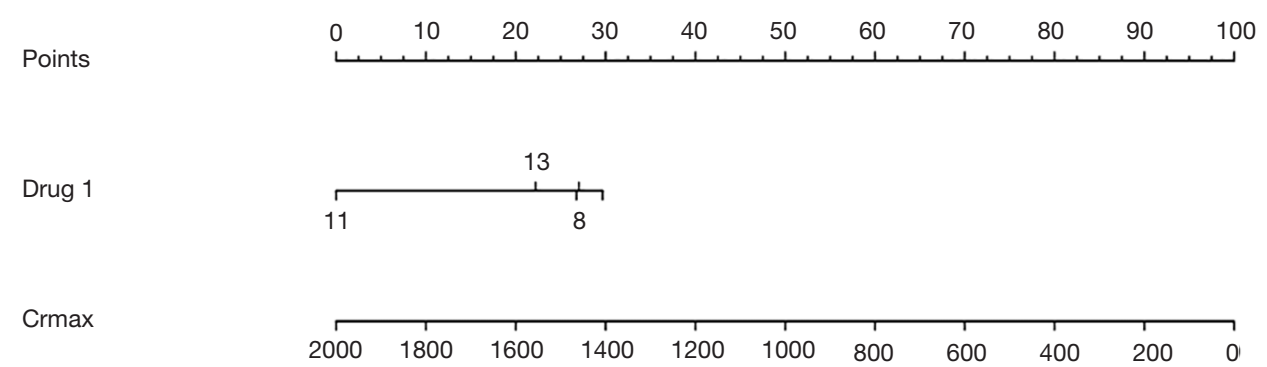

Total points

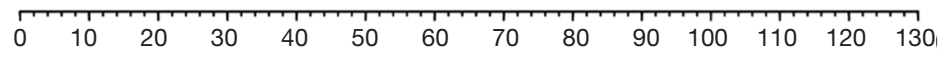

Recovery within 2 weeks

\begin{tabular}{lllllll}
\hline 0.1 & 0.2 & 0.3 & 0.4 & 0.5 & 0.6 & 0.7
\end{tabular}

Figure 5 Nomogram of predictors for predicting recovery within 2 weeks of drug-induced AKI. Drug $8=$ Antimicrobial agents, Drug $11=$ NSAIDs, Drug 13 = Chinese herbal medicine. AKI, acute kidney injury.

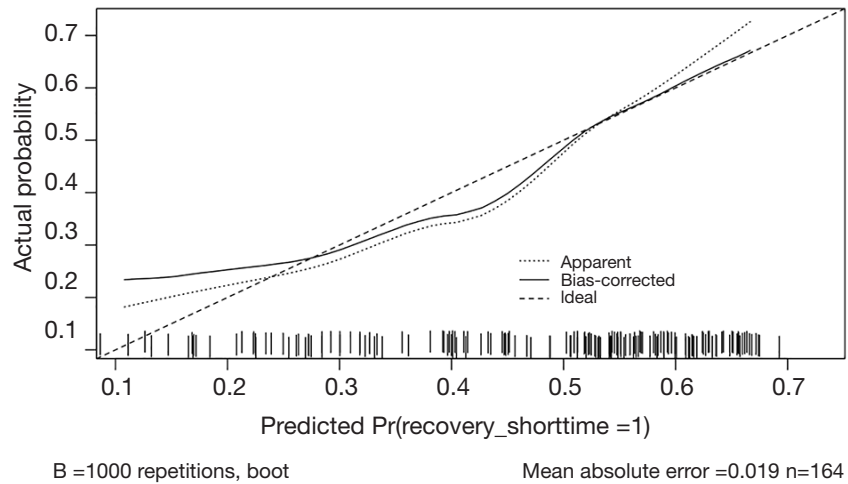

Figure 6 Calibration plots of internal validation.

more concomitantly administered drugs were involved in $66 \%$ of AKI cases. The most frequently implicated drug classes were antibacterial agents for systemic use (29.5\%), diuretics $(18.5 \%)$, agents acting on the renin-angiotensin system (16.3\%), antineoplastic agents (10.2\%), and antiinflammatory agents (5.4\%) and the drugs most frequently associated with AKI were antibacterial agents, diuretics, medications acting on the renin-angiotensin system, antineoplastic agents, and NSAIDs (10). In the present study, the most common causes of drug-induced AKI were antibiotics, NSAIDs, and Chinese herbal medicine, and the incidence of AKI was higher after the use of multiple drugs. This may be due to the increased awareness of AKI risk factors and the nephrotoxicity of some drugs such as vancomycin, diuretics, and ACEI, which made doctors selectively avoid the use of these drugs in high-risk groups. Our results showed that in AKI induced by antibiotics, vancomycin accounted for a small proportion, while betalactam antibiotics and combination drugs accounted for the vast majority (32.43\% and $33.78 \%$, respectively). The second most common drug to cause AKI were NSAIDs, which most patients reported taking out of the hospital for colds or pain relief. We suggest strengthening the public health education program alerting the community to the dangers of indiscriminate use of NSAIDs. Similarly, the use of Chinese herbal medicine, which was the third leading cause of AKI in this study, requires further scrutiny.

The risk factors for drug-induced AKI include age over 60 years, hypertension, diabetes history, alcohol history, and extracellular volume depletion $(21,22)$. In most studies, age was considered the most important risk factor for druginduced AKI. Pierson-Marchandise et al. (10) found that the mean age at which patients develop AKI was 68.7 15 .0-year and Feest et al. (7) showed that there was a progressive, agedependent, three- to eight-fold increase in the incidence of community-acquired AKI in patients over the age of 60 -year. Further, in a Japanese study, Kitano et al. (23) showed that patients in the 70-79-year age group were the most affected of all age groups. In our research, the average age of patients with drug-induced AKI was 51.1 16.4 -year, and $67.1 \%$ of patients were under 60 years of age, which is younger than that reported in other studies.

Furthermore, we found that the age distribution of AKI patients caused by different drugs was different. 
The average age of NSAIDs induced AKI patients in our study was 46 years, which is younger than those reported internationally (21). The average age of patients with AKI caused by antibiotics was 56 years, and by Chinese herbal medicine was 63 years. These differences in age distribution may be related to the medication habits of patients of different age groups.

The main pathological manifestations of druginduced renal injury can be divided into injury affecting the vasculature, glomerulus, renal tubules, and renal interstitium (24). Tubular injury, including acute tubular injury/necrosis, is usually caused by chemotherapeutic drugs, antimicrobial agents, and other medications, such as iron-chelating agents (25). Acute crystalline nephropathy is usually caused by antiviral agents (acyclovir, indinavir, atazanavir), antibacterial agents (sulfonamides, ciprofloxacin), methotrexate, triamterene, ascorbic acid, and sodium-phosphate purgatives $(26,27)$. Interstitial disease, including acute interstitial nephritis (AIN), is usually caused by antimicrobial agents, NSAIDs, selective cyclooxygenase inhibitors, proton pump inhibitors, and many other medications (25). Baker showed that a drug-induced etiology was the most common cause of AIN, presenting in 91 of 128 patients (28).

Vascular injury, including thrombotic microangiopathy, is usually caused by anti-angiogenesis drugs, gemcitabine, mitomycin $\mathrm{C}$, interferon, calcineurin inhibitors, $\mathrm{m}$-TOR inhibitors, thienopyridines, quinine, and oxymorphone (Opana) ER. Vasculitis is usually caused by propylthiouracil, infliximab, cocaine-containing levamisole, and atheroemboli, usually caused by anticoagulants (25). Glomerular injury, including the membranous type, is usually caused by NSAIDs, gold, bucillamine, and penicillamine, and lupus-like glomerulonephritis is usually caused by methyldopa, hydralazine, procainamide, and quinidine (29). Minimal change disease is usually caused by NSAIDs, lithium, interferon, pamidronate, vaccinations, and focal segmental glomerulosclerosis, usually caused by lithium and pamidronate, interferon, and sirolimus $(24,30)$.

In our research, the main renal pathological manifestations of drug-related AKI were AIN, allergic interstitial nephritis, and renal tubular epithelial injury (76.47\%). However, there was no specific correlation between the pathological manifestations of drug-induced renal injury and the causal agents. IgA, IgM, C3, C1q, and other immune complexes are deposited to different degrees in different renal pathologies, but further studies are needed to clarify the clinical significance.
Excessive intake of the Chinese herbal medicine aristolochic acid is a major cause of aristolochic acid nephropathy and Balkan nephropathy, a chronic tubulointerstitial disease with a high frequency of urothelia atypia (31). However, in our study, the 6 Chinese herbal medicine induced AKI patients who underwent renal biopsy were shown not to have aristolochic acid nephropathy. While the reasons for this are unclear, it may simply indicate the medication those patients used contained only small amounts of aristolochic acid.

Inherent features of patients such as age and sex have been identified as vital risk factors that increase the morbidity rate of drug-induced AKI patients (32). Previous research has suggested that the duration of $\mathrm{AKI}$ is associated with its prognosis $(11,12)$. However, there are few studies on renal recovery from AKI, especially on the recovery time and its factors. Our study showed that $72.4 \%$ of patients recovered completely, $1.8 \%$ of patients recovered partially, and $3.5 \%$ of patients did not recover, and age and gender were not risk factors for recovery. Although some patients were not followed up after discharge because their creatinine levels were decreasing, a graph of the renal function recovery rate over time showed about $90 \%$ of patients had kidney function recovery in 3 months. Furthermore, we established a nomogram to identify factors that predict recovery within two weeks of drug-induced AKI based on the maximum serum creatinine levels and the type of drug causing renal injury to help clinicians determine patients' prognosis in the early stage AKI.

There are limitations to this study, including its retrospective design and conduct in a single center. Multicenter prospective studies are required to validate the results fully. Also, some patients were treated in local hospitals where drugs were supplied using only "conventional doses." The dosage and duration of drug therapy is important and may affect the analysis of factors affecting the prognosis of AKI although the type of drugs inducing AKI is still of importance.

\section{Conclusions}

The three main contributors to drug-induced AKI were antimicrobial agents, NSAIDs, and Chinese herbal medicines. Of the antibacterial agents, the most implicated types were cephalosporins, acyclovir, clindamycin, azithromycin, and levofloxacin, but not aminoglycosides. The age distribution of those using these three different drugs is also significantly different. Allergic interstitial 
nephritis, interstitial nephritis, and tubular epithelial cell injury were the main pathological manifestations of druginduced AKI, but there was no specific correlation between the pathological manifestation and the drug that caused it. The novel predictive nomogram could predict recovery within 2 weeks in drug-induced AKI patients according to the type of drug and the maximum SCr.

\section{Acknowledgments}

We thank Dr. Qi Qian in the Division of Nephrology and Hypertension, Department of Medicine, Mayo Clinic College of Medicine (200 First Street SW Rochester, MN55905) for constructive instruction and comments on the design of the present study.

Funding: This work was supported by grants from the National Nature Science Foundation of China (No. 81770752, No. 81670621), and the Nature Science Foundation of Zhejiang Province (No. LY16H050001).

\section{Footnote}

Reporting Checklist: The authors have completed the STROBE reporting checklist. Available at http://dx.doi. org/10.21037/atm-20-3826

Data Sharing Statement: Available at http://dx.doi. org/10.21037/atm-20-3826

Conflicts of Interest: All authors have completed the ICMJE uniform disclosure form (available at http://dx.doi. org/10.21037/atm-20-3826). The authors have no conflicts of interest to declare.

Ethical Statement: The authors are accountable for all aspects of the work in ensuring that questions related to the accuracy or integrity of any part of the work are appropriately investigated and resolved. The study was conducted in accordance with the Declaration of Helsinki (as revised in 2013). The study was approved by the Research Ethics Committee of the First Affiliated Hospital, College of Medicine, Zhejiang University (No. 2019-1363). All patients provided written informed consent.

Open Access Statement: This is an Open Access article distributed in accordance with the Creative Commons Attribution-NonCommercial-NoDerivs 4.0 International License (CC BY-NC-ND 4.0), which permits the non- commercial replication and distribution of the article with the strict proviso that no changes or edits are made and the original work is properly cited (including links to both the formal publication through the relevant DOI and the license). See: https://creativecommons.org/licenses/by-nc-nd/4.0/.

\section{References}

1. Schetz M, Dasta J, Goldstein S, et al. Drug-induced acute kidney injury. Curr Opin Crit Care 2005;11:555-65.

2. Mehta RL, Pascual MT, Soroko S, et al. Spectrum of acute renal failure in the intensive care unit: the PICARD experience. Kidney Int 2004;66:1613-21.

3. Ali T, Khan I, Simpson W, et al. Incidence and outcomes in acute kidney injury: a comprehensive population-based study. J Am Soc Nephrol 2007;18:1292-8.

4. Hoste EA, Bagshaw SM, Bellomo R, et al. Epidemiology of acute kidney injury in critically ill patients: the multinational AKI-EPI study. Intensive Care Med 2015;41:1411-23.

5. Coca SG, Singanamala S, Parikh CR. Chronic kidney disease after acute kidney injury: a systematic review and meta-analysis. Kidney Int 2012;81:442-8.

6. Xue JL, Daniels F, Star RA, et al. Incidence and mortality of acute renal failure in Medicare beneficiaries, 1992 to 2001. J Am Soc Nephrol 2006;17:1135-42.

7. Feest TG, Round A, Hamad S. Incidence of severe acute renal failure in adults: results of a community based study. BMJ 1993;306:481-3.

8. Groeneveld AB, Tran DD, van der Meulen J, et al. Acute renal failure in the medical intensive care unit: predisposing, complicating factors and outcome. Nephron 1991;59:602-10.

9. Uchino S, Kellum JA, Bellomo R, et al. Acute renal failure in critically ill patients: a multinational, multicenter study. Jama 2005;294:813-8.

10. Pierson-Marchandise M, Gras V, Moragny J, et al. The drugs that mostly frequently induce acute kidney injury: a case - noncase study of a pharmacovigilance database. $\mathrm{Br} \mathrm{J}$ Clin Pharmacol 2017;83:1341-9.

11. Brown JR, Kramer RS, Coca SG, et al. Duration of acute kidney injury impacts long-term survival after cardiac surgery. Ann Thorac Surg 2010;90:1142-8.

12. Han SS, Kim S, Ahn SY, et al. Duration of acute kidney injury and mortality in critically ill patients: a retrospective observational study. BMC Nephrol 2013;14:133.

13. Perez MJ, Briz O. Bile-acid-induced cell injury and protection. World J Gastroenterol 2009;15:1677-89. 
14. Personett HA, Kayhart BM, Barreto EF, et al. Renal Recovery following Liposomal Amphotericin B-Induced Nephrotoxicity. Int J Nephrol 2019;2019:8629891.

15. Chawla LS, Bellomo R, Bihorac A, et al. Acute kidney disease and renal recovery: consensus report of the Acute Disease Quality Initiative (ADQI) 16 Workgroup. Nat Rev Nephrol 2017;13:241-57.

16. Kellum JA, Sileanu FE, Bihorac A, et al. Recovery after Acute Kidney Injury. Am J Respir Crit Care Med 2017;195:784-91.

17. Závada J, Hoste E, Cartin-Ceba R, et al. A comparison of three methods to estimate baseline creatinine for RIFLE classification. Nephrol Dial Transplant 2010;25:3911-8.

18. Liaño F, Felipe C, Tenorio MT, et al. Long-term outcome of acute tubular necrosis: a contribution to its natural history. Kidney Int 2007;71:679-86.

19. Marcum ZA, Amuan ME, Hanlon JT, et al. Prevalence of unplanned hospitalizations caused by adverse drug reactions in older veterans. J Am Geriatr Soc 2012;60:34-41.

20. Handler SM, Cheung PW, Culley CM, et al. Determining the incidence of drug-associated acute kidney injury in nursing home residents. J Am Med Dir Assoc 2014;15:719-24.

21. Cao YL, Tian ZG, Wang F, et al. Characteristics and clinical outcome of nonsteroidal anti-inflammatory druginduced acute hepato-nephrotoxicity among Chinese patients. World J Gastroenterol 2014;20:13956-65.

22. Lee TH, Lee CC, Ng CY, et al. The influence of acute kidney injury on the outcome of Stevens-Johnson syndrome and toxic epidermal necrolysis: The prognostic value of KDIGO staging. PLoS One 2018;13:e0203642.

Cite this article as: Cui Y, Yang Y, Lei W, Lang X, Chen J. The clinicopathological features of drug-induced acute kidney injury-a single-center retrospective analysis. Ann Transl Med 2021;9(5):400. doi: 10.21037/atm-20-3826
23. Kitano A, Motohashi H, Takayama A, et al. ValacyclovirInduced Acute Kidney Injury in Japanese Patients Based on the PMDA Adverse Drug Reactions Reporting Database. Ther Innov Regul Sci 2015;49:81-5.

24. Paueksakon P, Fogo AB. Drug-induced nephropathies. Histopathology 2017;70:94-108.

25. Perazella MA, Luciano RL. Review of select causes of drug-induced AKI. Expert Rev Clin Pharmacol 2015;8:367-71.

26. Perazella MA, Markowitz GS. Drug-induced acute interstitial nephritis. Nat Rev Nephrol 2010;6:461-70.

27. Blank ML, Parkin L, Paul C, et al. A nationwide nested case-control study indicates an increased risk of acute interstitial nephritis with proton pump inhibitor use. Kidney Int 2014;86:837-44.

28. Baker RJ, Pusey CD. The changing profile of acute tubulointerstitial nephritis. Nephrol Dial Transplant 2004;19:8-11.

29. Markowitz GS, Bomback AS, Perazella MA. Drug-induced glomerular disease: direct cellular injury. Clin J Am Soc Nephrol 2015;10:1291-9.

30. Markowitz GS, Nasr SH, Stokes MB, et al. Treatment with IFN-\{alpha\}, -\{beta\}, or -\{gamma\} is associated with collapsing focal segmental glomerulosclerosis. Clin J Am Soc Nephrol 2010;5:607-15.

31. Yang B, Xie Y, Guo MJ, et al. Nephrotoxicity and Chinese Herbal Medicine. Clinical Journal of the American Society of Nephrology 2018;13:1605-11.

32. Kane-Gill SL, Goldstein SL. Drug-Induced Acute Kidney Injury: A Focus on Risk Assessment for Prevention. Crit Care Clin 2015;31:675-84. 